\title{
NEURAL PATHWAY CONSTRAINTS IN THE MOTOR INNERVATION OF THE CHICK HINDLIMB FOLLOWING DORSOVENTRAL ROTATIONS OF DISTAL LIMB SEGMENTS ${ }^{1}$
}

\author{
VIRGINIA WHITELAW ${ }^{*, 2}$ AND MARGARET HOLLYDAY ${ }^{\ddagger}, 3$ \\ ${ }^{*}$ Department of Biophysics and Theoretical Biology and $\ddagger$ Department of Pharmacological and Physiological Sciences, \\ The University of Chicago, Chicago, Illinois 60637
}

Received August 11, 1982; Revised December 27, 1982; Accepted January 17, 1983

\begin{abstract}
Several studies have demonstrated that motor axons can discriminate between dorsally and ventrally derived muscles. In this paper we present evidence that (1) the pathway axons take in the limb constrain their access to either dorsally or ventrally derived muscles, and therefore (2) the axon's ability to discriminate between dorsal and ventral is expressed already at the level of pathway selection into the limb.

Surgically manipulated hindlimbs were produced consisting of a normal host thigh connected to a dorsoventrally rotated calf or to rotated and duplicated donor limb segments. The limb rotations were done distal to the level at which axons select a dorsally or ventrally destined pathway through the limb, such that at the level of the rotation, axons in each nerve were confronted with the opposite-from-normal set of muscles. In this situation, the relative influence of pathway availability versus dorsal/ventral muscle recognition could be assessed.

The innervation of rotated limb segments was, in all cases, opposite from normal. Motoneurons which normally innervate dorsal muscles innervated ventrally derived muscles that had been rotated into a dorsal position. Likewise, normally ventrally destined axons served dorsal muscles in the rotated segments. Thus, motor axons did not alter their distal path to reach their normal set of muscles.

While these results do not rule out intrinsic dorsal/ventral differences between muscles, they do demonstrate that muscle surface recognition is not necessary to account for dorsal/ventral discrimination in the innervation of normal, supernumerary, or duplicated limbs, nor is it sufficient to account for the innervation of rotated limb segments. These results also indicate that pathway guidance cues are an important influence on innervation patterns.
\end{abstract}

The developmental mechanisms underlying chick limb innervation can be inferred from experimental situations in which the normal spatial arrangements between motoneurons and targets are disrupted. The systematic behavior of motoneurons in various experimental situations provides clues as to the organizing principles which direct their projection.

In the previous paper (Whitelaw and Hollyday, 1983b) we presented evidence suggesting that growth cues as-

\footnotetext{
' This work was supported by National Institutes of Ilealth Grant NS-14066 to M. H. V. W. was supported by United States Public Health Service Grants GM-07183, GM-07839, and NS-14066. We thank Dr. Paul Grobstein for valuable discussions and for help in writing this paper.

${ }^{2}$ Present address: Bell Laboratories, Naperville, IL 60566.

${ }^{3}$ To whom correspondence should be sent at Department of Pharmacological and Physiological Sciences, University of Chicago, 947 East 58th Street, Chicago, IL 60637.
}

sociated with the limb plexuses influence the pattern of motor projection along the proximodistal axis. For example, motor axons associated with the crural plexus behaved as if they were constrained to choose among muscle targets in the first limb segment (i.e., the host thigh); they never grew farther distal even when a second thigh was grafted at the level of the second limb segment. Only motor axons in the peroneal and tibial nerves from the sciatic plexus projected beyond the first limb segment. The systematic misinnervation of duplicated limb segments demonstrated that motor axons grew no farther distally in duplicated limbs than they do in normal limbs. Thus, the proximodistal position of a duplicated muscle was important in determining which subset of motor axons had access to it.

Despite the observed mismatch along the proximodistal axis, we found that when motor axons innervated a novel muscle, it was derived from the same precursor 
muscle mass as their normal target; for example, if they would normally serve a dorsally derived muscle, their mismatched target would also be dorsally derived. This is the same result that was obtained in studies on supernumerary limbs (Hollyday et al., 1977; Hollyday, 1981; Lance--Jones and Landmesser, 1981b). In both situations, the thigh/calf recognition could be overridden, but the motor axons still discriminated between dorsal and ventral muscles.

In the present study we examine the extent to which this apparent dorsal/ventral discrimination could be the consequence of the available pathways restricting access to a given subset of limb muscles. Alternatively, there could be intrinsic differences among dorsal and ventral muscle surfaces which are influential in attracting or permitting innervation from a given subset of motor axons.

A direct way of assessing dorsal/ventral discrimination, namely by dorsoventral rotations of the limb bud, has been reported by two different groups with opposite results. Ferguson (1978) reported that despite the rotation, motoneurons displayed their characteristic preference for dorsally or ventrally derived muscles. Medially lying motor pools, for example, innervated ventrally derived muscles, despite the latters' aberrant positions. Summerbell and Stirling (1981), on the other hand, found that most of the time, medially lying motoneurons aberrantly innervated dorsally derived muscles (which had been rotated into a ventral position). Laterally lying motoneurons innervated ventrally derived muscles. In a minority of cases, the normal innervation pattern was obtained (as in Ferguson, 1978).

A relevant difference between the rotation experiments in which axons innervated their normal limb region and those in which they did not may well have been the proximodistal level of the rotation. The wing rotations done by Summerbell and Stirling (1981) were at the level of the shoulder or elbow. Unlike in Ferguson's (1978) experiments, the limb girdle was not consistently rotated in these operations (R. V. Stirling, personal communication). Consequently, in most embryos, the rotated tissue was distal to the point of plexus formation and axonal entry into the limb. Thus, motoneurons may have been comitted to aberrantly innervating the rotated tissue by virtue of having selected pathways leading to their appropriate muscle mass at the unrotated shoulder.

The findings of the two rotation studies can be reconciled if it is assumed that motoneurons are constrained to aberrantly innervate limb muscles if they enter the limb via their normal pathway and then encounter dorsoventrally rotated tissue. On the other hand, if they reach rotated tissue before they enter the limb, they can select their appropriate pathway into the limb and innervate their normal muscles. This hypothesis was tested in the present study by dorsoventral limb segment rotations at the level of the knee; i.e., distal to the point of nerve entry into the limb. At the time motor axons first grow into the limb, the dorsally destined and ventrally destined calf nerves are in the distal part of the thigh, spatially separated. A rotation at this level confronts each nerve with the opposite-from-normal set of muscles. In this manipulated situation, the relative importance of dorsal/ventral muscle recognition versus pathway cues can be assessed.

\section{Materials and Methods}

All operations were performed on White Leghorn chick embryos, staged according to the system of Hamburger and Hamilton (1951). Surgery was performed prior to nerve ingrowth into the limb bud, as described previously (Whitelaw and Hollyday, 1983a).

The dorsoventral rotation operations were performed on stage (St) 20 to 22 embryos. The distal half of the host's right leg bud was removed, leaving presumptive thigh precursor tissue intact. The distal tip of the limb bud from comparably staged donors was removed and fixed to the host's truncated leg. Both the dorsoventral and anteroposterior axes of the graft were reversed relative to the host's leg. A schematic representation of the operation is shown in Figure 1. The resultant limbs consisted of either a normal host thigh connected to a rotated calf, or a partial host leg connected to rotated and duplicated graft segments. Surgery was performed on approximately 70 embryos.

Histological analysis. Motor pools serving rotated and, in some cases, rotated and duplicated muscles were mapped in St 36-38 embryos using intramuscular injections of horseradish perixodase (HRP), as described previously (Whitelaw and Hollyday, 1983a). Injections were made either in ovo or in organ culture. The experimental limbs were carefully reconstructed, as before, to identify the musculature, the peripheral innervation pattern, and, where appropriate, the HRP injection site.

\section{Results}

Characteristics of St 36-38 rotated limbs. Dorsoventrally rotated limbs were characterized by their skeletal elements. All limbs used in this study consisted of a normal host thigh connected to a dorsoventrally rotated graft. The only limbs included were those in which the graft calf was rotated 150 to $180^{\circ}$ relative to the host limb axes. Two such limbs stained for cartilage are shown

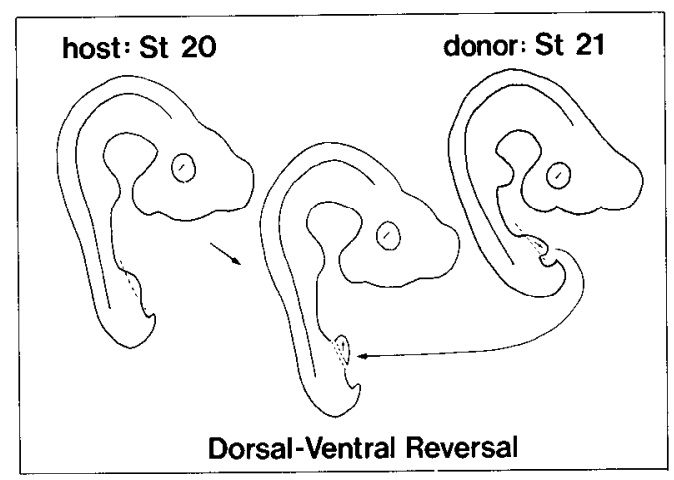

Figure 1. Schematic drawing of the dorsoventral rotation operation. The distal half of a St 21 donor was removed, rotated $180^{\circ}$, and fitted to a truncated host leg. The graft was held in place by the surrounding membranes. By varying the amount of host tissue removed and donor tissue transferred, limbs were produced consisting of either a normal host thigh connected to a dorsoventrally rotated calf and foot or a partial host limb connected to rotated and duplicated limb segments. 


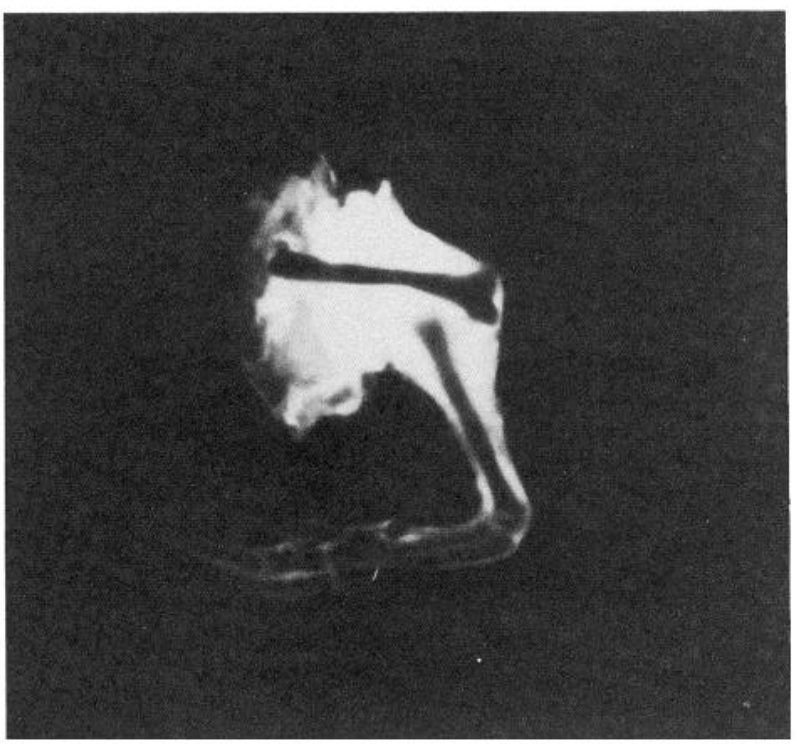

a

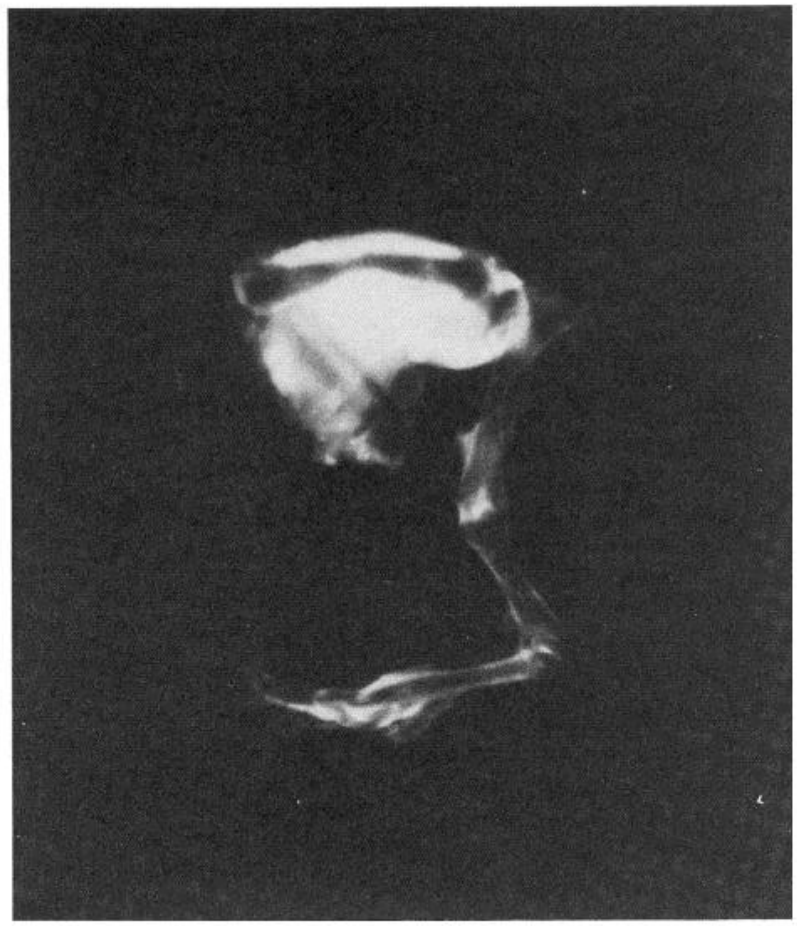

b

Figure 2. Whole mount limbs with dorsoventral segment rotations stained for cartilage. $a$, The limb is composed of a normal host thigh and a dorsoventrally rotated calf and foot. $b$, A serially duplicated and dorsoventrally rotated limb of the type thigh-thigh-calf-foot, where the distal three segments are in reverse orientation relative to the host. Note the extensive muscle atrophy in these segments. Both limbs are from St 36 embryos and are shown 5 times their actual size. in Figure 2. The limb in Figure $2 a$ consists of a dorsoventrally rotated calf and foot connected to a normal host thigh, but no leg segments are duplicated. Figure $2 b$ shows a limb with both rotated and duplicated segments. The motor innervation results from these two types of limbs are presented in this order.

HRP injections of dorsoventrally rotated calves. HRP was injected into either dorsal or ventral muscles of the rotated calves. Injections into dorsal calf muscles of three embryos resulted in labeling of the medial cluster of motoneurons in all cases. Motoneurons in these positions normally serve ventral calf muscles. In contrast, ventral calf injections in six embryos consistently labeled cells in caudo-intermediate positions which normally serve dorsal calf muscles. An example of the labeling pattern following a ventral calf injection of such a limb is shown in Figure 3. Nearly all of the label resides in the pools which normally serve the dorsal calf muscles, tibialis anterior and peroneus longus. The medially lying motor pools which normally serve the injected muscles are shown in shading on the control side of the spinal cord (from Hollyday, 1980). Very few or no labeled cells are seen in this region on the experimental side.

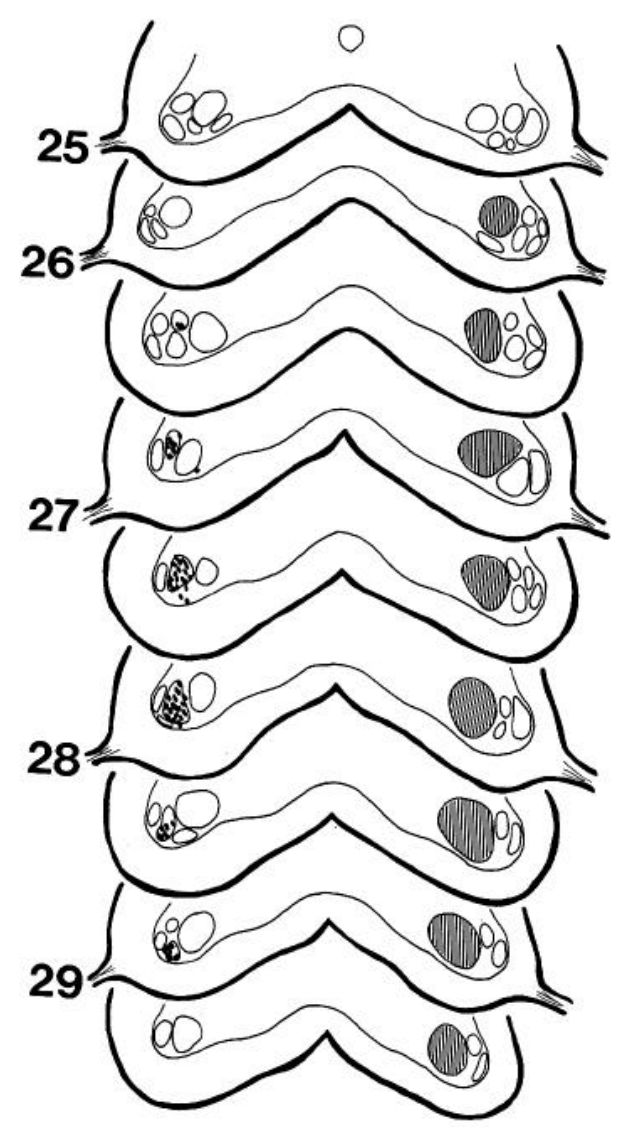

Figure 3. Reconstruction of the distribution of labeled cells following an HRP injection of ventral muscles in a dorsoventrally rotated calf. The normal motor pools for the injected muscles are shaded (from Hollyday, 1980). The labeled motoneurons are not in a corresponding position on the experimental side. Rather, they are in caudo-intermediate motor pools which normally innervate dorsal calf muscles. 
TABLE I

Distribution and averaged percentages of labeled cells following HRP injections of dorsoventrally rotated calves ${ }^{a}$

\begin{tabular}{|c|c|c|c|c|c|c|c|c|c|c|c|c|}
\hline $\begin{array}{l}\text { muscle } \\
\text { group } \\
\text { injected }\end{array}$ & $\begin{array}{l}\text { number } \\
\text { of } \\
\text { cases }\end{array}$ & $\begin{array}{l}\text { number of } \\
\text { labeled } \\
\text { cells/case }\end{array}$ & $\begin{array}{l}\text { average } \\
\text { number of } \\
\text { labeled } \\
\text { cells/case }\end{array}$ & position & \multicolumn{7}{|c|}{ distribution of labeled cells } & $\begin{array}{c}\text { percent in } \\
\text { normal } \\
\text { motor pool } \\
\text { region }\end{array}$ \\
\hline ventra] & \multirow{3}{*}{6} & 50 & \multirow{3}{*}{95} & I & & & 17.2 & 26.7 & 31.8 & 14.4 & .9 & \multirow[t]{3}{*}{7.4} \\
\hline calf & & 99 & & $\mathrm{~L}$ & & & .7 & .5 & .2 & .2 & & \\
\hline & & 183 & & & & & & & & & & \\
\hline \multirow[b]{2}{*}{$\begin{array}{c}\text { dorsal } \\
\text { calf }\end{array}$} & \multirow[b]{2}{*}{3} & 90 & \multirow[b]{2}{*}{209} & M & & & & 6.5 & 32.1 & 45.6 & 15.0 & \multirow[b]{2}{*}{.7} \\
\hline & & 134 & & I & & & & .3 & .4 & & & \\
\hline
\end{tabular}

${ }^{a}$ Heavy lines indicate the position of motor pools innervating ventral and dorsal calf muscles in normal animals. M, medial; I, intermediate; $L$, lateral.

The results from all nine dorsoventral calf rotations are summarized in Table I. The distribution of label is schematized on the grid as described previously (Whitelaw and Hollyday, 1983b). The normal positions of motor pools serving the injected muscles are outlined with heavy lines. For both classes of injections, very few of the labeled cells are in these regions.

The average number of labeled cells resulting from injections of rotated calves (average for dorsal injections: 209 cells/case; average for ventral injections: 95 cells/ case) is somewhat less than the numbers obtained following comparably sized injections of unrotated second limb segments (see Whitelaw and Hollyday, 1983b). Interestingly, the average number of labeled cells following dorsal injections is twice that found following ventral injections. This is opposite to the pattern found in normal embryos and is consistent with the fact that, normally, there are many more motoneurons in the medial cluster, which supplies ventral calf muscles, than there are in the intermediate pools, which supply dorsally derived calf muscles.

HRP injections of dorsoventrally rotated and duplicated segments. HRP injections were also made into two types of duplicated limbs in which either an entire hindlimb (thigh-calf-foot) was grafted in reversed dorsoventral orientation onto a host thigh, or a calf and foot were rotated and grafted to the calf of the host.

The results of duplicated and rotated graft injections were in many respects similar to those presented in the previous paper (Whitelaw and Hollyday, 1983b) for injections of duplicated limb segments. In all embryos, when injections were made into grafted tissue in the position of the second limb segment, normal calf motor pools were labeled (six cases). Following injections of third limb segments, label was characteristically sparse and peripherally located in the medial cluster (three cases); cells in these positions normally supply intrinsic foot muscles. Injections of farther distal segments resulted in no label in the lateral motor column (LMC).
The one sense in which the results of duplicated reversals differed from those of serial duplications was that motor pools which normally innervated dorsal muscles projected instead to ventral muscles and vice versa. Data have already been presented above for dorsoventral mismatch among calf motor pools serving rotated second limb segments. The results of injections into rotated and duplicated second limb segments agree with these findings; i.e., medially lying motoneurons aberrantly innervated dorsally derived muscles and intermediately positioned motoneurons aberrantly innervated ventral muscles. Injections of duplicated and rotated third limb segments revealed a similar mismatch among foot motor pools as described below.

In all three cases in which successful injections were made of a duplicated and rotated calf in the position of the third segment, mismatch among presumptive foot motor pools resulted. An injection of the dorsal, distal calf mass resulted in label in the far medial periphery of the medial cluster, a region which normally serves ventral intrinsic foot muscles. Injections of ventral distal calf labeled peripheral-intermediate cells in the LMC in a region which normally serves dorsal foot muscles. The amount of label observed following these injections (average: 25 labeled cells/case) was comparable to that obtained following other injections of the third distal leg segment.

A sample reconstruction of a ventral distal calf injection is shown in Figure 4. On the control side of the LMC, shading indicates the far medial region in which labeled cells are found following ventral injections of unrotated duplicated calves (from Whitelaw and Hollyday, 1983b, Fig. 4). The position of labeled cells on the experimental side in this rotated and duplicated case is on the opposite side of the medial cluster; i.e., on the lateral border.

Thus, the finding of dorsoventral mismatch in the innervation of rotated third limb segments is consistent with the similar finding in rotated second limb segments (i.e., simple calf rotations). The finding of labeled cells at 
the peripheral border of the medial motoneuron cluster following injections of rotated third limb segments is consistent with what is found in unrotated third limb segments. The motor pool results of rotated and unrotated as well as duplicated and unduplicated limb injections are summarized for comparison in Table II. "I" and " $M$ " denote, respectively, "intermediate" and "medial" calf motor pool label; " $P$ " indicates peripheral label, normally associated with foot motor pools. As Table II makes clear, second limb segment injections label normal calf motor pools and third limb segment injections label peripheral pools. Normally medial pools innervate ventral muscles and intermediate pools innervate dorsal muscles; however, following injections into dorsoventrally rotated segments, the opposite pattern of label is found.

Nerve reconstructions from dorsoventrally rotated grafts. Reconstructions of the nerve-branching patterns in the dorsoventrally rotated grafts corroborated the motor pool finding of reversed innervation. In these limbs, as in normal legs, the peroneal and tibial nerves diverged from one another near the distal end of the host

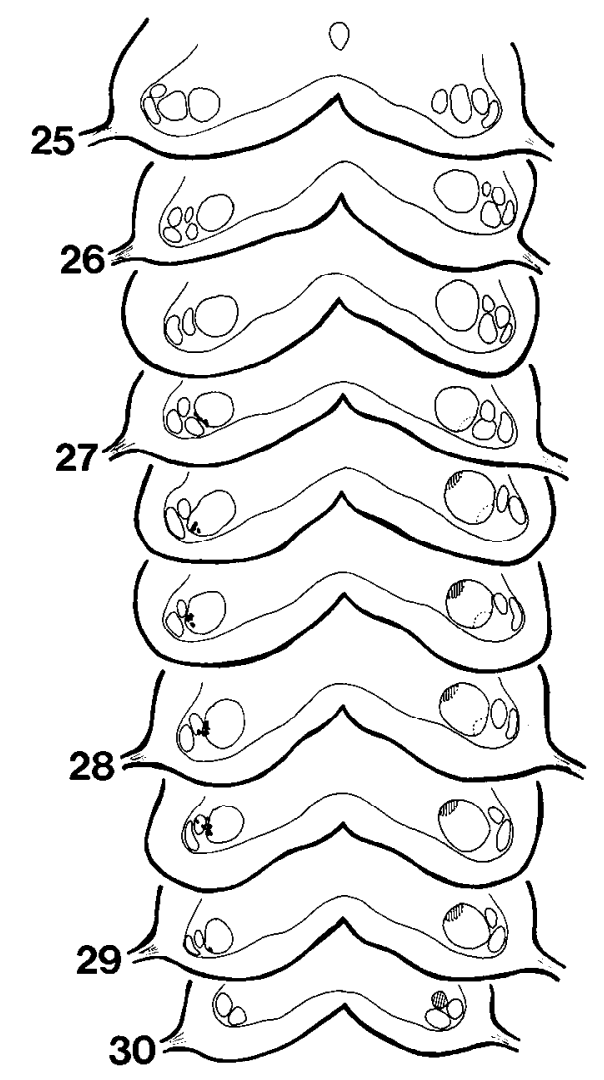

Figure 4. Reconstruction of the distribution of labeled cells for an $\mathrm{HRP}$ injection of ventral muscles in a dorsoventrally rotated and duplicated (third segment) calf. The shaded motor pool indicates the position of labeled motoneurons following ventral muscle injections in duplicated (but not rotated) third segment calves (from Whitelaw and Hollyday, 1983b, Fig. 4). The position of labeled motoneurons in this duplicated and rotated case is on the opposite periphery of the medial cluster, in the region of a motor pool which normally serves a dorsal intrinsic foot muscle. This region is indicated by a broken line on the control side.
TABLE II

Comparison between the positions of labeled cells following HRP injections of second and third limb segments in normal, duplicated, and rotated limbs

\begin{tabular}{lcccccc}
\hline \multirow{2}{*}{ Type of Limb } & \multicolumn{4}{c}{ Injected Segment } \\
\cline { 2 - 3 } & \multicolumn{2}{c}{$\begin{array}{c}\text { Second Limb Seg- } \\
\text { ment }\end{array}$} & & \multicolumn{2}{c}{$\begin{array}{c}\text { Third Limb Seg- } \\
\text { ment }\end{array}$} \\
\cline { 2 - 3 } \cline { 5 - 7 } & \multicolumn{2}{c}{ Dorsal } & Ventral & & Dorsal & Ventral \\
\hline Normal leg & $\mathbf{I}^{a}$ & $\mathbf{M}$ & & P-I & P-M \\
Duplicated leg & $\mathbf{I}$ & $\mathbf{M}$ & & P-I & P-M \\
Dorsoventrally rotated leg & $\mathbf{M}$ & $\mathrm{I}$ & & P-M & P-I \\
\hline
\end{tabular}

"Abbreviations: I, intermediate; $\mathrm{M}$, medial; $\mathrm{P}$, peripheral.

thigh. In general, these two nerves were seen on opposite sides of the knee-like cartilage, which was the level of the dorsoventral rotation. 'This resulted in each nerve entering the opposite-from-normal set of muscles farther distally. The peroneal nerve, which normally innervates dorsal muscles, aberrantly served ventral muscles in these legs; the tibial nerve innervated dorsal muscles.

Although some dorsal and ventral muscles were innervated in all of the rotated calves examined, it did not appear that all of the muscles were innervated. In particular, some of the deep ventral muscles of the calf were often uninnervated and atrophied, and, in one case, despite the clear presence of the tibial nerve amidst dorsal calf muscles, most of them appeared extremely atrophic and no point of nerve entry into them could be found. All of the muscles in general were smaller than those in normal calves. Thus, the innervation of rotated calves could not properly be called a mirror image of the normal innervation pattern. The peroneal and tibial axons certainly did not go out of their way to find their normal targets, but neither did they seem to innervate all possible abnormal targets.

The innervation of muscles in rotated third limb segments was accomplished by characteristically small nerves. As in rotated second limb segments, branches of the peroneal nerve aberrantly innervated ventral muscles, while the tibial nerve served dorsal muscles. No nerve branches were evident beyond the third limb segment.

The limb reconstructions confirmed that the graft rotations were distal to the level at which the peroneal and tibial nerves diverged along different pathways. Evidently, once so committed to a pathway, the axons could not grow to their normal targets.

\section{Discussion}

Evidence for neural pathway constraints. By virtue of its association with a nerve or neural pathway, a motor axon's choice of muscle targets can be limited to either dorsally or ventrally derived muscles. Such is the conclusion from dorsoventral graft rotations in which limb segments were rotated distal to the level at which the nerves selected either a dorsal or a ventral pathway through them. The result was that dorsally derived muscles were aberrantly innervated by medially lying motoneurons, and ventrally derived muscles were aberrantly innervated by laterally positioned motoneurons. The normally dorsally serving peroneal nerve innervated ventral 
calf muscles; the tibial nerve served the dorsal calf. In rotated and duplicated third limb segments, the motor pools serving the dorsal and ventral muscles were in positions opposite from where they were found in unrotated third limb segments (Whitelaw and Hollyday, 1983b).

These results imply a proximal commitment on the part of axons to pathways which restrict access to the subset of muscles derived from either dorsal or ventral muscle mass. Once a dorsally or ventrally destined pathway is selected, the motor axons innervate a dorsal or ventral muscle, respectively. There does not seem to be an opportunity for axons to change pathways (i.e., from dorsal to ventral) once so committed. Even in experimental situations where novel pathways have been described (Hollyday, 1981; Lance-Jones and Landmesser, 1981b), they have been branches from dorsally or ventrally destined nerve trunks which remained in dorsally or ventrally derived muscles, respectively.

A consideration of pathway commitment suggests a relevant difference between the dorsoventral rotations reported here and those reported by Ferguson (1978); namely, the proximodistal level of the rotation. In the latter experiments, whole limb rotations were performed, including the hip girdle, and the result was normal motor pool organization (i.e., lateral pools innervated dorsal muscles; medial pools served ventral muscles). Assuming that these rotations were done proximal to the level at which axons were committed to certain pathways, then the axons could still select their normal pathways, despite the rotation, by whatever mechanism they normally use. On the other hand, when the rotation is distal to the level at which axons make their first pathway choice, they are evidently constrained to innervate the wrong set of muscles. This is the result not only in the present study, but also in the dorsoventral rotations reported by Summerbell and Stirling (1981).

These results do not rule out the possibility that there are intrinsic dorsal/ventral differences between muscles and that axons normally "prefer" one type or the other. But they do indicate that dorsoventral recognition at the level of the muscles is not necessary to account for the selective mismatch which has been described in the innervation of duplicated (Whitelaw and Hollyday, 1983b) and supernumerary (Hollyday, 1981) limbs. The explanation for our findings seems to be that each motor axon, on the basis of the position of its cell body in the motor column, becomes associated with one of several nerves entering the limb. Anterior thigh muscles are served from the crural plexus; posterior thigh, calf, and foot muscles are innervated from the sciatic plexus. Emerging from each plexus are separate nerves which exclusively serve either dorsally or ventrally derived muscles. Thus when a motor axon becomes associated with one of these pathways, it is in effect selecting for dorsal or ventral targets. The decision as to which pathway a motor axon joins is definitely not random as the medial and lateral motor axons consistently sort out from one another the same way. The fact that this decision cannot be reversed later in the limb argues that selective factors involved in dorsal/ventral recognition operate at the level of the plexus. To determine whether there is in addition a dorsal/ventral recognition which operates at the level of interaction with the muscles themselves requires a different type of experimental situation; i.e., one which can reveal whether motor axons free of substrate guidance cues can distinguish between different muscles.

Comparison to other manipulated situations. While previous studies have not explicitly focused on the role of the limb tissue in restricting axonal access to various groups of muscles, there is precedence for the idea that the limb directs the branching of nerves within it. For example, when forelimbs are transplanted so that they receive lumbar motor innervation, the peripheral nerve patterns are clearly forelimb-like, not hindlimb-like (Harrison, 1907; Detwiler, 1936; Hollyday, 1981). Moreover, supernumerary grafts have shown that both the type of plexus formed and the detailed pattern of nerve branching are determined by the type of limb and not by the segmental nerves (Morris, 1978; Hollyday, 1981).

The role of neural pathways in limiting the choice of target muscles provides a consistent interpretation of the results of several previously reported manipulations of this system. For example, following deletions of the rostral spinal segments which norrnally form the crural plexus, the muscles it would have normally served remained uninnervated (Lance-Jones and Landmesser, 1980a). The fact that axons associated with the sciatic plexus did not expand into this foreign territory agrees with the hypothesis that when a motor axon is associated with a given nerve pathway, it only has access to a subset of muscles. If those same spinal segments were rotated rostrocaudally (instead of deleted), normal motor innervation resulted (Lance-Jones and Landmesser, 1980b). In this rotated case, as in normal innervation, these segments formed a normal crural plexus and entered the anterior thigh via the normal pathways. The fact that normal innervation resulted is consistent with the idea that, once associated with the correct nerve pathway, a motor axon generally innervates its normal muscle. If, however, a long piece of spinal cord is rotated so that when motor axons reach the base of the limb, they are far from their normal plexus, then motor axons usually fail to innervate their normal targets (Lance-Jones and Landmesser, 1981b). These results as well as those in supernumerary limbs indicate that pathway availability is important in restricting axonal access to subsets of limb muscles. Pathway availability can account for both normal and mismatched patterns of innervation, depending on the experimental situation.

Thus, the importance of pathway availability in organizing the projection of motor axons is demonstrated not only by the dorsoventral graft rotations, but in supernumerary limbs and in other manipulated situations as well. It would be an oversimplification, however, to suggest that pathway availability is the entire story. For example, in embryos with supernumerary or anteriorly shifted limbs or large spinal cord reversals, some muscles are innervated by means of an aberrant route (Hollyday, 1981; Lance-Jones and Landmesser, 1981b). Moreover, in embryos with limb segment deletions, there is a selectivity in the innervation of the remaining segment which cannot be entirely explained by the presence or absence of pathways in the limb tissue. Thus, a combination of organizing principles is implicated in the development of 
limb innervation (Hollyday and Grobstein, 1981). The nature of such a synthesis is suggested by experimental situations in which different mechanisms apparently dominate. One possible synthesis drawing upon the results of this paper and the two preceding ones (Whitelaw and Hollyday, 1983a, b) is presented below.

Developmental mechanisms involved in chick limb innervation. Several developmental mechanisms have been proposed as organizers of specific neuronal connections (Hollyday, et al., 1977; Stirling and Summerbell, 1977; Jacobson, 1978; Bennett et al., 1980; Landmesser, 1980; Lance-Jones and Landmesser, 1980b, 1981b). They cover a spectrum with regard to the amount of information required to organize the projection. At one extreme are mechanisms which posit no intrinsic individuation among afferents and targets, but instead emphasize the organizing capacity of the timing or spatial arrangement of afferent arrival at target structures (Bunt et al., 1978; Bennett et al., 1980). As discussed previously, such mechanisms cannot account for the selective innervation of thigh and calf muscles evident in partial deletion studies. Moreover, the full-limb dorsoventral rotation studies in which motoneurons innervate their normal targets (Ferguson, 1978) provide evidence for a dorsal/ventral selectivity at the level of the plexus. This selectivity cannot be accounted for by the serendipitous spatial arrangement or timing of axon arrival at the limb bud. Agerelated characteristics among the motor axons and the maintenance of their relative spatial relationships may well have some role in organizing the motor projection, but they are clearly not the only mechanisms responsible.

Similarly, a model of limb innervation based on target recognition cannot account for the motor projection (Hollyday, 1980). Target recognition hypotheses posit chemical individuation among the afferents and targets which provide a basis for selective interactions between them. A simple hypothesis of this sort might posit a chemically specified nerve moving freely through the limb, sampling the chemically specified musculature until it found a "best match." Such a hypothesis would predict that motor pools would serve their normal muscles regardless of where those muscles occurred, or whether they occurred twice. This fails to describe the innervation of duplicated limbs (Whitelaw and Hollyday, 1983b) or dorsoventrally rotated calves. In these manipulated situations, motor pools did not always innervate their normal targets. Rotated dorsal muscles were served by normally ventrally destined nerves; duplicated thighs were innervated by calf motoneurons; duplicated calves were served by presumptive foot motor pools, and many muscles were not innervated at all.

Thus, a simple target recognition hypothesis fails in its prediction of innervation in these and other manipulated situations. Its basic assumptions of individuated neurons and specific cues in the limb bud are likely to be true, as there is good evidence for selectivity in this system. But the other assumption of freely mobile nerves is almost certain to be wrong. Indeed, our studies provide good evidence that neural pathways constrain nerve access to a given subset of limb muscles. Selective interactions may well be involved, however, in the motor axons's decision as to which neural pathway to enter and at which muscle to exit.
In the case of proximal muscles, pathway selection may be manifest at the level of the individual motor pool (Lance-Jones and Landmesser, 1981a). It seems unlikely, however, that pathway selection at the level of the plexus can entirely account for the normal pattern of limb innervation, especially for the projections to distal limb segments where axons destined for different targets follow common pathways. Additional pathway choices and developmental mechanisms are likely to be involved in patterning such projections.

In summary, the innervation of partial limbs demonstrates differential response on the part of motor axons to growth cues in the limb and suggests that competitive interactions may be important for determining which axons have access to a particular pathway. Limb segment duplications and rotations indicate that axonal growth can be influenced both by guidance cues at the point of pathway selection into the limb and by pathway opportunities and constraints located farther distally. The different experimental manipulations provide situations in which different organizing principles dominate the patterns of limb innervation. It seems likely that the projection pattern resulting in the case of normal development reflects the combined action of all of these influences.

From the results of the manipulations of the chick limb system one can formulate a possible interpretation of how the several mechanisms interact in the development of the motor projection. Early in development, before the axons have grown into the limb, the motor axons sort out at the plexus into several discrete nerves (Lance-Jones and Landmesser, 1981a). It is plausible that age- or position-dependent properties of the motoneurons mediate this consistent segregation. As the axons enter the limb from the plexus, they select pathways leading to either dorsal or ventral muscle mass. Proximal growth cues involved in pathway recognition may also delimit the subset of muscles to which each group of axons has access, as well as influence growth along the proximodistal axis. Within each nerve, the motor projection is generally continuous (neighboring motoneuron clusters in the spinal cord innervate neighboring muscles in the limb). This map continuity may result from the tendency of axons from neighboring motoneurons to recognize each other along their growth pathway and hence to project accordingly. As the axons traverse the limb along a particular pathway, competitive interactions based on target recognition may further direct local organization among the axons growing along a common path.

\section{References}

Bennett, M. R., D. F. Davey, and K. E. Uebel (1980) The growth of segmental nerves from the brachial myotomes into the proximal muscles of the chick forelimb during development. J. Comp. Neurol. 189: 335-357.

Bunt, J. S. M., T. J. Horder, and K. A. C. Martin (1978) Evidence that optic fibers regenerating across the goldfish tectum may be assigned termination sites on a "first-come, first-served" basis. J. Physiol. (Lond.) 276: 45-46P.

Detwiler, S. R. (1936) Neuroembryology: An Experimental Study, Macmillan Publishing Co., Inc., Reprint ed., Hafner Publishing Co., New York, 1964.

Ferguson, B. (1978) Effect of dorso-ventral limb rotations on the development of motor connections. Soc. Neurosci. Abstr. 
4: 111 .

Hamburger, V., and H. Hamilton (1951) A series of normal stages in the development of the chick embryo. J. Morphol. 88: 49-92.

Harrison, R. G. (1907) Experiments in transplanting limbs and their bearing upon the problems of the development of nerves. J. Exp. Zool. 4: 239-281.

Hollyday, M. (1980) Organization of motor pools in chick lumbar lateral motor column. J. Comp. Neurol. 194: 143-170.

Hollyday, M. (1981) Rules of motor innervation in chick embryos with supernumerary limbs. J. Comp. Neurol. 202: 439465.

Hollyday, M., and P. Grobstein (1981) Of limbs and eyes and neuronal connectivity. In Studies in Developmental Neuro. biology. Essays in Honor of Viktor Hamburger, W. M. Cowan, ed., pp. 188-217, Oxford University Press, New York.

Hollyday, M., V. Hamburger, and J. M. G. Farris (1977) Localization of motor neuron pools supplying identified muscles in normal and supernumerary legs of chick embryos. Proc. Natl. Acad. Sci. U. S. A. 74: 3582-3586.

Jacobson, M. (1978) Developmental Neurobiology, Ed. 2, Plenum Publishing Corp., New York.

Lance-Jones, C., and L. Landmesser (1980a) Motoneuron projection patterns in embryonic chick limbs following partial deletions of the spinal cord. J. Physiol. (Lond.) 302: 559-580.

Lance-Jones, C., and L. Landmesser (1980b) Motoneuron projection patterns in the chick hindlimb following early partial reversals of the spinal cord. J. Physiol. (Lond.) 302: 581-602.

Lance-Jones, C., and L. Landmesser (1981a) Pathway selection by chick lumbosacral motoneurons during normal development. Proc. R. Soc. Lond. Biol. 260: 1-18.

Lance-Jones, C., and L. Landmesser (1981b) Pathway selection by embryonic chick motoneurons in an experimentally altered environment. Proc. R. Soc. Lond. Biol. 260: 19-52.

Landmesser, L. (1980) The generation of neuromuscular specificity. Annu. Rev. Neurosci. 3: 279-302.

Morris, D. G. (1978) Development of functional motor innervation in supernumerary hindlimbs of the chick embryo. J. Neurophysiol. 41: 1450-1465.

Stirling, R. V., and D. Summerbell (1977) The development of functional innervation in the chick wing-bud following truncations and deletions of the proximo-distal axis. J. Embryol. Exp. Morphol. 41: 189-207.

Summerbell, D., and R. V. Stirling (1981) 'The innervation of dorso-ventrally reversed chick wings: Evidence that motor axons do not actively seek out their appropriate targets. J. Embryol. Exp. Morphol. 61: 233-247.

Whitelaw, V., and M. Hollyday (1983a) Thigh and calf discrimination in the motor innervation of the chick hindlimb following deletions of limb segments. J. Neurosci. 3: 1199-1215.

Whitelaw, V., and M. Hollyday (1983b) Position-dependent motor innervation of the chick hindlimb following serial and parallel duplications of limb segments. J. Neurosci. 3: 12161225. 